\title{
Proteomics and Phosphoproteomics Profiling of Drug-Addicted BRAFi-Resistant Melanoma Cells
}

\author{
Bohui Li, Xiangjun Kong, Harm Post, Linsey Raaijmakers, Daniel S. Peeper, and Maarten Altelaar*
}

Cite This: J. Proteome Res. 2021, 20, 4381-4392

Read Online

ACCESS | LWll Metrics \& More | 回 Article Recommendations

Supporting Information
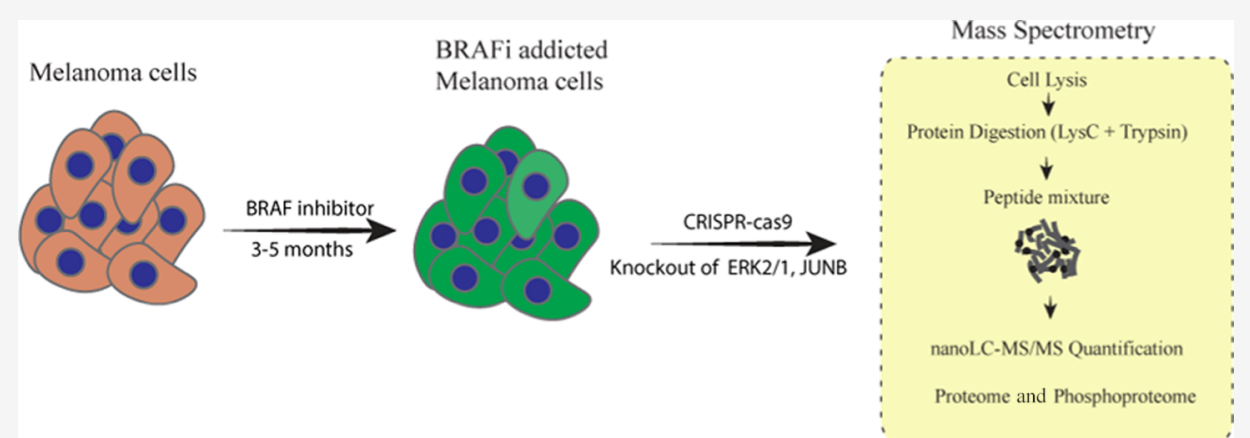

ABSTRACT: Acquired resistance to MAPK inhibitors limits the clinical efficacy in melanoma treatment. We and others have recently shown that $\mathrm{BRAF}$ inhibitor (BRAFi)-resistant melanoma cells can develop a dependency on the therapeutic drugs to which they have acquired resistance, creating a vulnerability for these cells that can potentially be exploited in cancer treatment. In drugaddicted melanoma cells, it was shown that this induction of cell death was preceded by a specific ERK2-dependent phenotype switch; however, the underlying molecular mechanisms are largely lacking. To increase the molecular understanding of this drug dependency, we applied a mass spectrometry-based proteomic approach on BRAFi-resistant BRAF ${ }^{\mathrm{MUT}}$ 451Lu cells, in which ERK1, ERK2, and JUNB were silenced separately using CRISPR-Cas9. Inactivation of ERK2 and, to a lesser extent, JUNB prevents drug addiction in these melanoma cells, while, conversely, knockout of ERK1 fails to reverse this phenotype, showing a response similar to that of control cells. Our analysis reveals that ERK2 and JUNB share comparable proteome responses dominated by reactivation of cell division. Importantly, we find that EMT activation in drug-addicted melanoma cells upon drug withdrawal is affected by silencing ERK2 but not ERK1. Moreover, transcription factor (regulator) enrichment shows that PIR acts as an effector of ERK2 and phosphoproteome analysis reveals that silencing of ERK2 but not ERK1 leads to amplification of GSK3 kinase activity. Our results depict possible mechanisms of drug addiction in melanoma, which may provide a guide for therapeutic strategies in drug-resistant melanoma.

KEYWORDS: melanoma, BRAF-resistant, proteomics, phosphoproteomics, ERK2/MAPK1, ERK1/MAPK3, EMT

\section{INTRODUCTION}

Oncogenic mutations that cause activation of BRAF occur regularly in melanoma, with approximately 40 to $60 \%$ of cutaneous melanomas carrying mutations in BRAF (e.g., BRAF-V600E). Such mutations lead to constitutive activation of downstream signaling through the RAF/MEK/ERK mitogen-activated protein kinase (MAPK) pathway, ${ }^{1}$ making BRAF an attractive target for antimelanoma therapy. Thus, small molecules (inhibitors) were designed to target the MAPK pathway, such as vemurafenib and dabrafenib, which are selective BRAF mutant inhibitors. ${ }^{2}$ Although these BRAF inhibitors (BRAFi's) showed a lot of potential in melanoma treatment, with remarkable response rates and overall survival, ${ }^{3}$ the clinical benefit is hindered by the rapid development of acquired resistance.

Many routes to the acquisition of BRAFi resistance are described, such as BRAF allele amplification or splice variants, ${ }^{4}$ reactivation of the MAPK pathway, and substitutive pathways. $^{5,6}$ The main mechanisms leading to MAPK reactivation and sustained ERK signaling involve alterations in BRAF, NRAS, MEK, and neurofibromin1 (NF1). ${ }^{7,8}$ The compensatory PI3K-mTOR cascade is the most commonly activated in drug-resistant melanoma, via gene mutation or deletion of PTEN, or the activation of receptor tyrosine kinases. ${ }^{9-11}$ Interestingly, several studies have shown that discontinued drug treatment in the resistant melanoma cells causes massive

Received: April 21, 2021

Published: August 3, 2021 
cell mortality, in other words, these resistant cells become addicted to the very drugs that initially served to eliminate them. $^{12-14}$

Typically, the BRAFi-addicted melanoma cells experience a transient cell-cycle slowdown, followed by cell death upon drug withdrawal. This specific phenotype induces pERK reactivation that upregulates p38-FRA1-JUNB-CDKN1A expression and slows down proliferation, and a robust $\mathrm{pERK}$ reactivation can result in DNA damage and parthanatos-related cell death. ${ }^{14}$ Moreover, ERK2, but not ERK1, was shown to be a "switch" in cancer drug addiction, since drug withdrawal-induced cell death in melanoma could be reversed by genetic inactivation of ERK2. ${ }^{12}$ Transcription factors such as JUNB, FRA1, and MITF were found to play key roles in such ERK2-dependent drug addiction switch, by reprogramming the ERK2-JUNBFRA1-MITF pathway. ${ }^{12}$

To systematically depict the alteration of the proteome and phosphoproteome involved in drug-addicted melanoma, we present a proteomic and phosphoproteomic study of BRAFiaddicted melanoma cells (i.e., $451 \mathrm{Lu}$ cell line) in response to BRAFi withdrawal. To shed light on the role of ERK1, ERK2, and JUNB in response to drug withdrawal, we genetically silenced these genes separately by CRISPR-Cas9, in these BRAFi-addicted melanoma cells, followed by systematic proteomic and phosphoproteomic profiling.

\section{MATERIALS AND METHODS}

\section{Cell Culture and Colony Formation}

The BRAF inhibitor dabrafenib, the MEK inhibitor trametinib, and the ERK inhibitor SCH772984 were purchased from Selleck Chemicals. 451Lu cells were obtained from J. Villanueva (The Wistar Institute). The A375, Mel888, and A101D cells were from the Peeper laboratory cell line stock. Cells were routinely tested for mycoplasma contamination and authenticated by STR profiling (Promega). Next cells were cultured in DMEM supplemented with $9 \%$ fetal bovine serum (Sigma), plus 100 units per $\mathrm{mL}$ penicillin and $0.1 \mathrm{mg} \mathrm{mL}^{-1}$ streptomycin (Gibco). To generate BRAFi- or BRAFi + MEKiresistant cells, parental drug-sensitive cells were exposed to increasing concentrations of BRAFi dabrafenib (from 0.01 to 5 $\mu \mathrm{M}$ ) or BRAFi dabrafenib + MEKi trametinib (from $0.01 \mu \mathrm{M}$ $+0.001 \mu \mathrm{M}$ to $0.5 \mu \mathrm{M}+0.05 \mu \mathrm{M})$ for $3-5$ months. The drugresistance phenotype of cells was verified by colony formation assay. The generation of ERK2, ERK1, or JUNB knockout pools and colony formation assay to determine cell viability in the presence or absence of BRAFi were performed as previously described. ${ }^{12}$ Resistant cells were stained with crystal violet ( $1 \%$ in $50 \%$ methanol) and photographed at day 0 with drug and day 3 without drug. The relative colony area was calculated using the plugin "ColonyArea" in ImageJ. ${ }^{15}$ Samples were collected at day 0 with drug and day 1 and day 3 without drug for further mass spectrometry analysis.

\section{Immunoblotting}

Immunoblotting was performed as previously described. ${ }^{16}$ The BRAFi + MEKi-resistant (Mel888 BMR, A101 BMR, and A375 BMR) or BRAFi-resistant (451Lu BR) cells were treated with the corresponding inhibitors (BRAFi + MEKi for BMR cells and BRAFi for BR cells), with an ERK inhibitor, or drug was withdrawn, all for $24 \mathrm{~h}$. Cells were harvested and total cell lysates were prepared and submitted for immunoblotting. The antibody for pirin was obtained from BD Bioscience.

\section{Protein Digestion}

Cells were lysed, reduced, and alkylated in lysis buffer [ $1 \%$ sodium deoxycholate, $10 \mathrm{mM}$ tris(2-carboxyethyl)-phosphine hydrochloride, $40 \mathrm{mM}$ chloroacetamide, and $100 \mathrm{mM}$ Tris], $\mathrm{pH}$ 8.0, supplemented with a protease inhibitor (cOmplete mini EDTA-free, Roche) and phosphatase inhibitor (PhosSTOP, Merck) and heated for $5 \mathrm{~min}$ at $95{ }^{\circ} \mathrm{C}$, followed by sonication with a Bioruptor Plus (Diagenode) for 15 cycles of $30 \mathrm{~s}$. Samples were diluted 1:10 with $50 \mathrm{mM}$ ammoniumbicarbonate (AMBIC), $\mathrm{pH}$ 8.0, and digested with Lys-C (1:200 ratio $\mathrm{w} / \mathrm{w}, \mathrm{Wako})$ and trypsin $(1: 50$ ratio $\mathrm{w} / \mathrm{w}$, Merck) at 37 ${ }^{\circ} \mathrm{C}$, overnight. Digestion was quenched by acidification to $2 \%$ formic acid and followed by desalting using 1cc Sep-Pak C18 cartridges (Waters Corporation).

\section{Phosphopeptide Enrichment}

Phosphorylated peptides were enriched using Fe(III)-NTA cartridges (Agilent technologies) in an automated fashion with the AssayMAP Bravo Platform (Agilent Technologies) as described. ${ }^{16}$ Briefly, $\mathrm{Fe}(\mathrm{III})-\mathrm{NTA}$ cartridges were primed using $0.1 \%$ TFA in ACN and equilibrated with loading buffer $(80 \%$ ACN $/ 0.1 \%$ TFA). Samples were dissolved in loading buffer and loaded onto the cartridge. The columns were washed with loading buffer, and the phosphorylated peptides were eluted with $1 \%$ ammonia directly into $10 \%$ formic acid and dried down.

\section{Mass Spectrometry: RP-nanoLC-MS/MS}

Resuspended peptides were subjected to LC-LC MS/MS using an Agilent 1290 Infinity coupled to an Orbitrap Q Exactive HF mass spectrometer (Thermo Scientific, Bremen, Germany) using a $160 \mathrm{~min}$ gradient for the full proteome samples and a $100 \mathrm{~min}$ gradient for the phospho-enriched samples. Peptides were first trapped (Dr Maisch Reprosil C18, $3 \mu \mathrm{m}, 2 \mathrm{~cm} \times 100 \mu \mathrm{m}$ ) before being separated on an analytical column (Agilent Poroshell EC-C18, $2.7 \mu \mathrm{m}, 50 \mathrm{~cm} \times 75 \mu \mathrm{m}$ ). Trapping was performed for $5 \mathrm{~min}$ in solvent A $(0.1 \mathrm{M}$ acetic acid in water) at $5 \mu \mathrm{L} \mathrm{min}{ }^{-1}$. The LC flow during the gradient

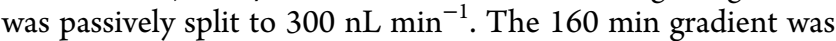
as follows: $13-44 \%$ solvent B ( $0.1 \mathrm{M}$ acetic acid in $80 \% \mathrm{ACN})$ for $152 \mathrm{~min}, 44-100 \%$ for $3 \mathrm{~min}$, and $100 \%$ for $4 \mathrm{~min}$. The 100 min gradient was as follows: $9-36 \%$ solvent B (0.1 M acetic acid in $80 \% \mathrm{ACN}$ ) for $93 \mathrm{~min}, 36-100 \%$ for $3 \mathrm{~min}$, and $100 \%$ for $4 \mathrm{~min}$. The mass spectrometer was operated in the data-dependent mode. Full-scan MS spectra from $m / z$ 3751600 were acquired at a resolution of 60,000 at $\mathrm{m} / z 200$ after accumulation to a target value of $3 \times 10^{6}$. Up to 15 most intense precursor ions were selected for fragmentation for the full proteome samples and up to 12 most intense precursor ions were selected for the phosphopeptide-enriched samples. HCD fragmentation was performed at a normalized collision energy of 27.

\section{Data Processing}

All raw MS files were searched using MaxQuant software (version 1.5.8.3). ${ }^{17}$ MS/MS spectra were searched by Andromeda against a reviewedHomo sapiensdatabase (UniProt, 20,197 entries, August, 2016). The parameters are as follows: trypsin digestion, maximum of two missed cleavages, cysteine carbamidomethylation as fixed modification, oxidized methionine, protein N-terminal acetylation, and serine/threonine/ tyrosine phosphorylation (for the phosphoproteome data analysis only) as variable modifications. Mass tolerance was set to 4.5 and $20 \mathrm{ppm}$ for the MS1 and MS2, respectively. The 
A

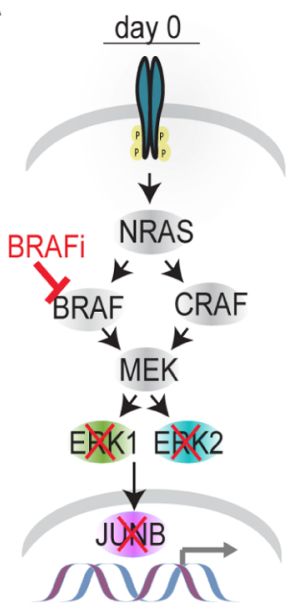

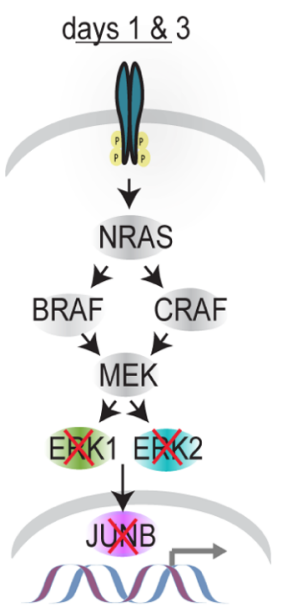

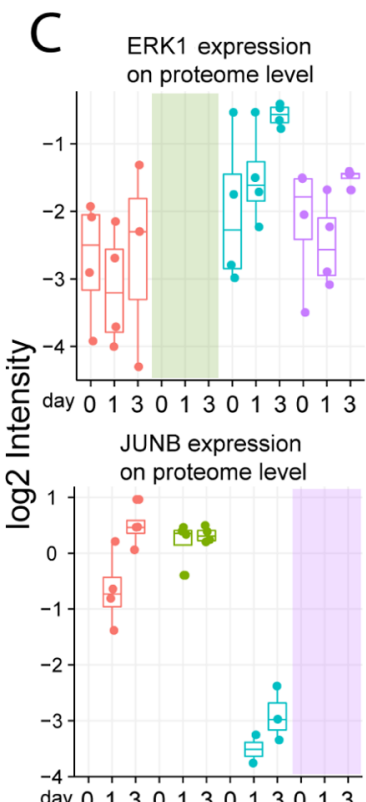

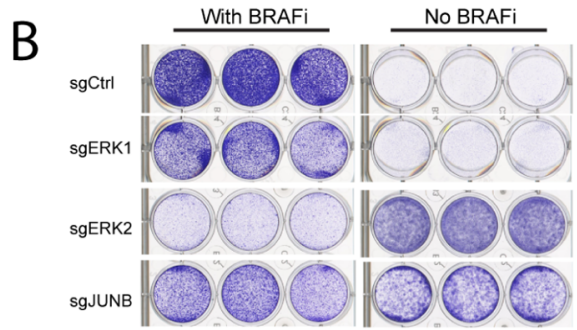
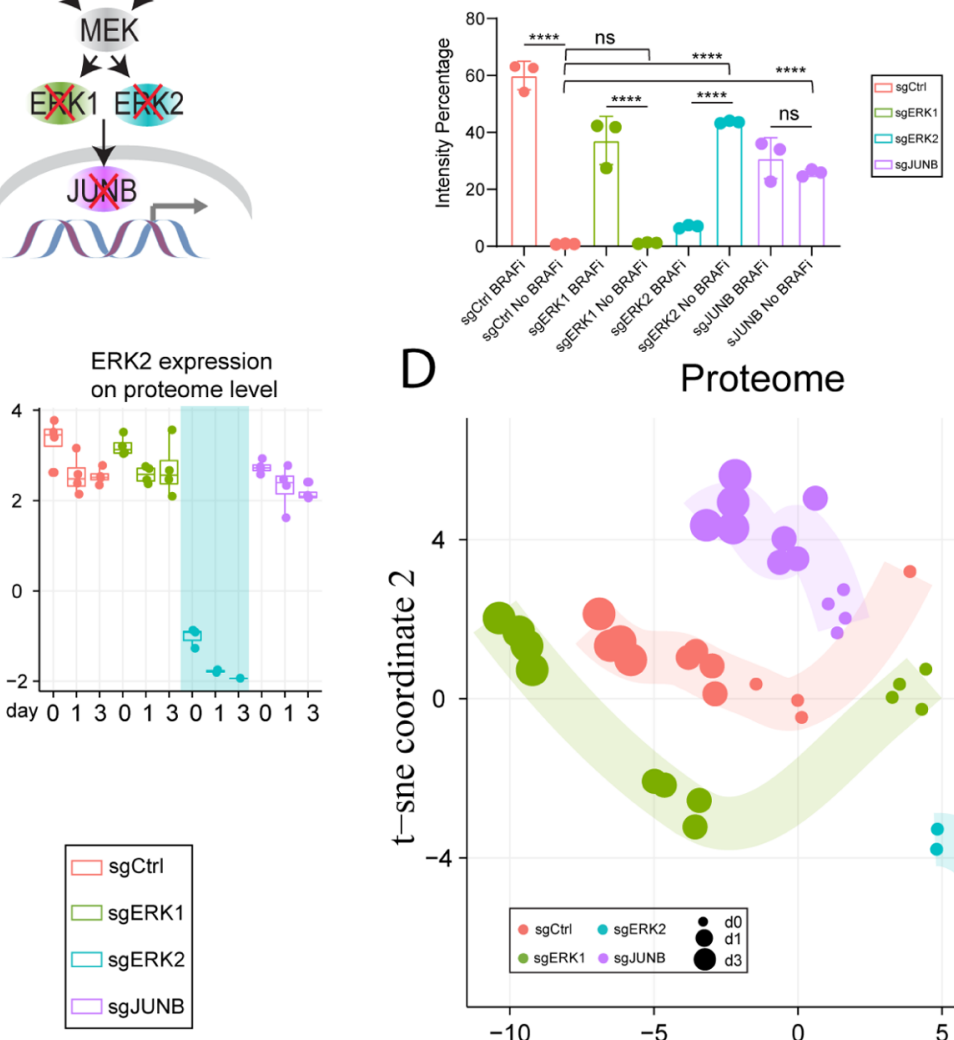

$\mathrm{D}$

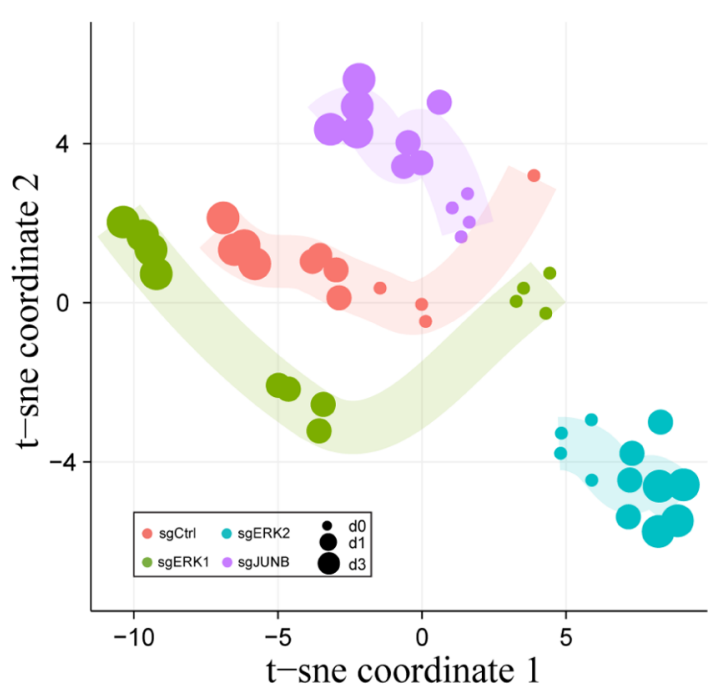

Figure 1. Phenotype and proteomic trajectory. (A) Separate knockout of ERK1, ERK2, and JUNB in BRAF inhibitor (dabrafenib)-addicted 451Lu cells, with drug on (day 0) and drug off (days 1 and 3). (B top) Control and 3 CRISPR-Cas9 knockout cells were cultured with or without BRAF inhibitor dabrafenib, followed by staining for the live cells. (B bottom) Relative colony area was calculated using ImageJ. Statistical differences were analyzed by one-way ANOVA with Tukey post-hoc testing (****, $p<0.0001$, error bars denote \pm SD). (C) Box plot illustrating protein abundance of each knockout gene. The points are not shown when the abundance is below detectability. (D) t-SNE plot, based on proteome profiling, shows the trajectory of how different cells proceeded upon drug withdrawal.

protein and PSM false discovery rate (FDR) were set to $1 \%$. Peptide identifications by MS/MS were transferred between runs to replace missing values for quantification, with a $0.7 \mathrm{~min}$ window after retention time alignment.

\section{Data Analysis and Statistics}

All data were analyzed under the R-3.5.1 environment ${ }^{18}$ and Microsoft Excel. Raw intensities extracted from MaxQuant were first $\log 2$-transformed and then normalized by the median of replicates for label-free quantification. We assumed that missing values were below detectability; thus, sample minimums were used to replace missing values. Furthermore, $\mathrm{t}$-distributed stochastic neighbor embedding $(\mathrm{t}-\mathrm{SNE})^{19}$ was employed to assess the reproducibility of the experiments within biological replicates $(n=4)$. To obtain the differentially expressed (DE) proteins between ERK1-silenced cells and nonsilenced cells, we fitted two linear regression models, where the first linear regression model indicates the observed differences between conditions that associate with gene silencing, which was fitted for each protein using the "lm" function in $\mathrm{R}$. The second linear regression model indicates no difference between the conditions. Next, the likelihood ratio test (LRT) was used to compare these two models to get the differential $p$-value for each protein using the "anova" function with the parameter "test = "LRT"” in R. The log2-fold change in the intensity for each protein was calculated by $\log 2$ (As/ $\mathrm{An})$, where "As" is the mean protein abundance in the Erk1silenced cells, and the "An" is the mean abundance in the control cells. The same analyses were performed to obtain the DE proteins between ERK2-silenced cells and nonsilenced cells, and JUNB-silenced cells and nonsilenced cells. Next, the DE proteins with FDR-corrected $p$-value $<0.05$ and $\log 2$-fold change $>1$ were further used for unsupervised clustering using 
A Differential Proteins in sgERK2

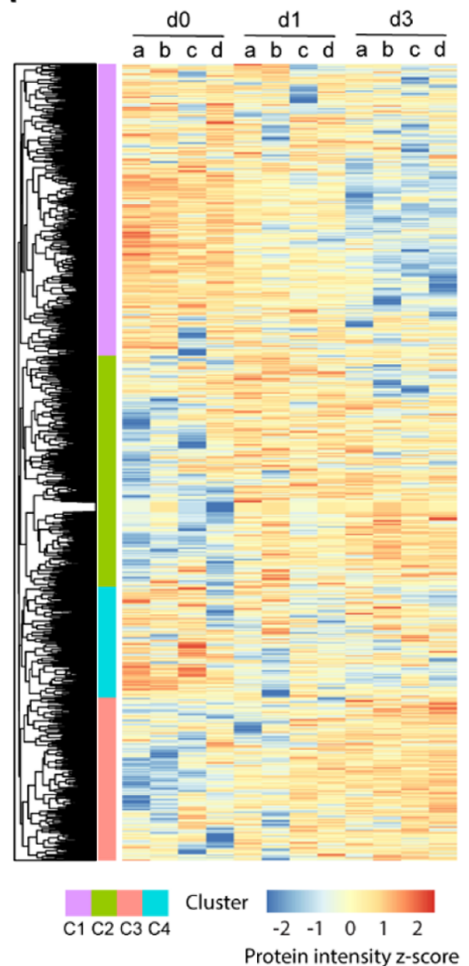

Differential Proteins in sgERK1

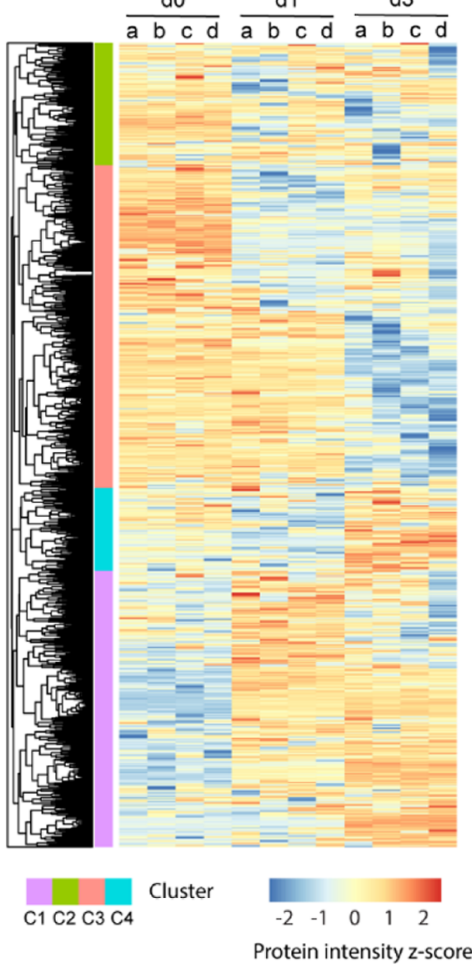

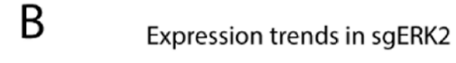

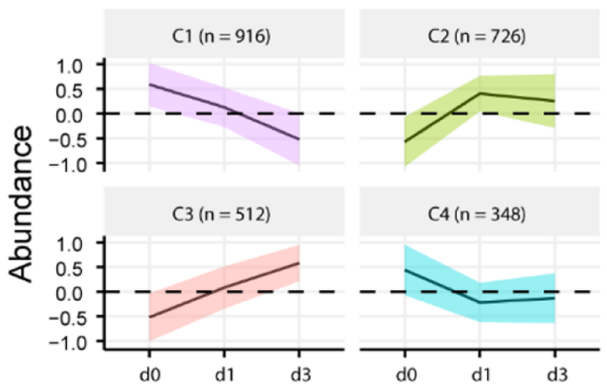

C Expression trends in sgERK1

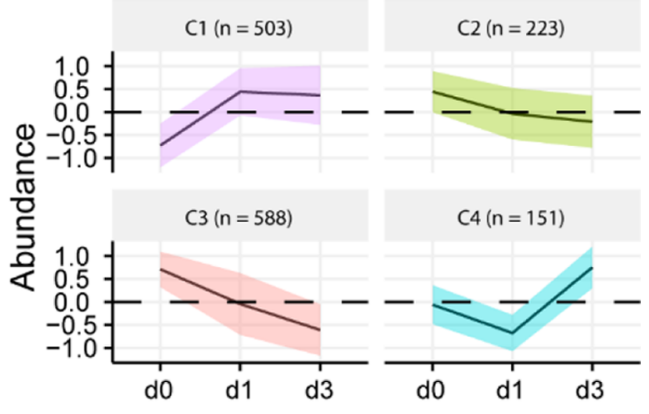

D Functional annotation with differential proteins in sgERK2

E Functional annotation with differential proteins in sgERK1
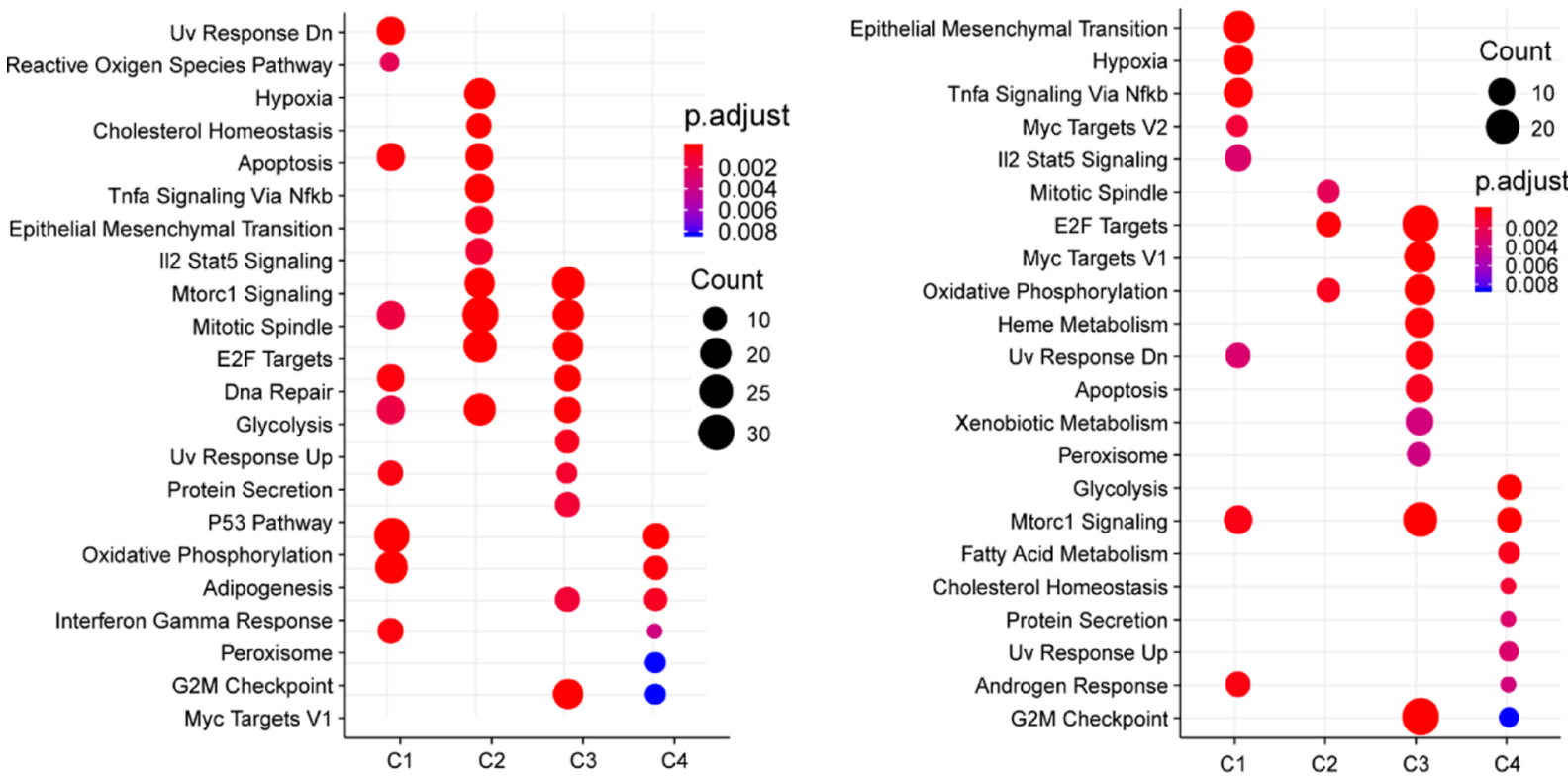

Figure 2. Proteome response in drug-addicted cells upon drug withdrawal. (A) Heat map of differential expression proteins in ERK2 knockout (left) and ERK1 knockout (right), constructed using unsupervised hierarchical clustering upon drug on (day 0 a, b, c, and d) and drug off (day 1 and day 3 a, b, c, and d), which shows four distinct expression patterns. (B,C) Average abundance trends for clusters in sgERK2 and sgERK1, shading denotes $\pm 1 \mathrm{SD}$. (D,E) Hallmark enrichment according to proteins in the corresponding cluster.

Euclidean as distance measurement and then cut into four clusters based on expression patterns over time. Subsequently, DE proteins in each cluster were used for the hallmark enrichment analysis in Metascape (a gene annotation \& analysis resource) online platform. ${ }^{20}$ Following this, the top 10 enriched (min overlap: 3; $p$-value cutoff: 0.01 ; min enrichment: 1.5) hallmarks for each cluster were used to show the enrichment difference.

\section{Transcription Factor (Regulator) Enrichment}

The RegEnrich R package that integrates proteome expression profiling, transcription factors (regulators), and WGCNA coexpression networks was performed to define the key transcription factors. ${ }^{21,22}$ Briefly, it involves three steps: (1) construction of data-driven coexpression networks using proteome profiles in control and each sgRNA cells; (2) deducing genes of interest (i.e., using differentially changed 
protein lists to obtain a TF-connected subnetwork); and (3) referring importance to TFs by Fisher's exact test. An enrichment score for TFs was given by incorporating the exhibited significance of differential expression $\left(p_{\mathrm{D}}<0.05\right)$ and significance of enrichment $\left(p_{\mathrm{E}}<0.05\right)$. The overall scores of TFs were calculated by

$$
\text { score }=\operatorname{norm}\left(-\log \left(p_{\mathrm{E}}\right)\right)+\operatorname{norm}\left(-\log \left(p_{\mathrm{D}}\right)\right)
$$

where $\operatorname{norm}(x)=\frac{x-\min (x)}{\max (x)-\min (x)}$.

\section{Kinase Activity}

In our phosphoproteome profiling, we quantified 22,117 phosphosites, of which 15,124 had a localization probability over 0.75 , mapping to 4070 proteins. A single sample-based public R package InKA (Integrative Inferred Kinase Activity) that integrates kinase-centric (e.g., kinome and activation loop) and substrate-centric (e.g., PhosphoSitePlus and NetworKIN) information was applied to infer active kinases. ${ }^{23}$ Following this, the top 20 activated kinases in each sample was used to show the activity difference.

\section{Data Availability}

All mass spectrometry proteomics data have been deposited to the ProteomeXchange Consortium via the PRIDE ${ }^{24}$ partner repository with the data set identifier PXD026557.

\section{RESULTS AND DISCUSSION}

\section{Drug Addiction Phenotype Switching}

In this study, we sought to increase our knowledge on the dynamic response of the proteome and associated signaling networks of drug-addicted melanoma cells in the presence and absence of drug, using a (phospho)proteomics approach. ERK1, ERK2, and JUNB genes were separately silenced by CRISPR-Cas9 in dabrafenib (BRAF inhibitor)-addicted 451Lu cells (Figure 1A). These cells carry out a BRAF V600E mutation and an MEK1 K57N-activating mutation. ${ }^{25}$ Interestingly, ceasing drug administration in drug-addicted melanoma cells triggered massive cell death in the control condition (Figure 1B). As observed before, ${ }^{12} \mathrm{KO}$ of ERK2 can drastically reverse this phenotype, that is, ERK2 knockout prevents drug addiction and cell death upon drug withdrawal. Conversely, inactivation of ERK1 failed to prevent drug addiction and resulted in severe cell death upon drug withdrawal, comparable to the control situation (Figure 1B). Loss of JUNB showed comparable results to ERK2 $\mathrm{KO}$ but to a lesser extent. These results suggest that the loss of ERK2 or JUNB in BRAFiaddicted melanoma cells could prevent the drug addiction phenotype.

To gain insights into resistance mechanisms to BRAFiaddicted melanoma, we employed a label-free quantitative (phospho)proteomics approach. Briefly, cells were collected at day 0 (with drug) and day 1 and day 3 (without drug) and lysed, and subsequently, the protein extracts were in-solutiondigested by LysC/trypsin and analyzed by liquid chromatography-tandem mass spectrometry (nanoLC-MS/MS) on a high-resolution mass spectrometer (Q-Exactive HF). The quantified protein abundances show the depletion of ERK1, JUNB, and ERK2, compared to the control (Figure 1C). Interestingly, sgCtrl, sgERK1, and sgERK2 cells show JUNB activation upon drug withdrawal, which is consistent with previous results. $^{12}$
In total, 5720 proteins with at least two unique peptides were quantified (Table S1A), on which we performed $t$-SNE analysis. Here, we found that cells within biological replicates cluster tightly together, while cells that carry different gene KOs followed distinct trajectories over time (Figure 1D). Of note, the cells in which ERK2 was depleted followed an opposite trajectory to the other cells over time. These results together suggest that ERK2 knockout considerably influences the proteome profiles of BRAFi-addicted melanoma cells.

Proteome Response in Drug-Addicted Cells upon Drug Withdrawal

To compare proteome changes between sgERK2 and sgCtrl upon drug exposure and withdrawal, 2502 significantly changed proteins between sgERK2 and sgCtrl were identified using the "anova" function in R with 5\% FDR and >two fold change. Next, an unsupervised hierarchical clustering method was applied to identify protein clusters with similar expression trends (Figure 2A). A total of four expression patterns were identified, with C2 (cluster 2) and C3 (cluster 3) displaying upregulated and $\mathrm{C} 1$ (cluster 1) and $\mathrm{C} 4$ (cluster 4) downregulated patterns (Figure 2A,B) upon drug removal. Pearson correlation using $2502 \mathrm{DE}$ proteins revealed that samples upon drug withdrawal showed higher correlation (Figure S1A).

Clusters C2 and C3 contain upregulated proteins, where C2 contains 726 proteins that increase expression immediately after drug removal and are stable afterward and C3 contains 512 proteins, showing proteins that continuously increase in expression upon drug withdrawal. Proteins in these clusters show the strongest enrichment related to mTORC1 signaling, cytokine (IL-2 and TNF- $\alpha$ ) signaling, cell division, and DNA repair processes (Figure 2D). Together, these results highlight that drug withdrawal in melanoma cells with inactivated ERK2 leads to reactivation of cell division, dominating the molecular processes observed. Among the downregulated clusters (Figure 2B), C1 showed continuous downregulation upon drug withdrawal and $\mathrm{C} 4$ includes proteins downregulated at day 1 and remaining stable afterward. Hallmark analysis showed oxidative phosphorylation as the most predominant pathway in both clusters (Figure 2D).

To understand the different molecular mechanisms involved between sgERK2 and sgERK1 cells, we next analyzed significantly changing proteins of ERK1-depleted cells upon drug withdrawal compared to sgCtrl. This analysis identified four clusters with similar expression patterns, with more than half of the proteins showing downregulation after drug withdrawal (Figure 2A right panel and 2C). Pearson correlation with 1465 differential proteins showed higher correlation within bioreplicates, where the correlation between biological replicates generally exceeded 0.9 (Figure S1B). Upregulated proteins upon drug removal in $\mathrm{C} 1$ and $\mathrm{C} 4$ enriched in epithelial mesenchymal transition (EMT) and cytokine and mTORC1 signaling (Figure 2E). Clusters C2 and C3, showing downregulation of proteins, significantly enriched in the cell cycle (E2F targets and G2M checkpoint), followed by energy metabolism and apoptosis (Figure 2E).

EMT is characterized by the loss of typical epithelial histologic features and gain of mesenchymal characteristics. ${ }^{26}$ These changes enhance cell migratory capacity and increase invasiveness, which enable the transition from melanoma in situ to aggressive, invasive melanoma. ${ }^{27}$ Our previous work showed that cells lacking ERK2, but not ERK1, failed to undergo the EMT-like changes following drug withdrawal. 

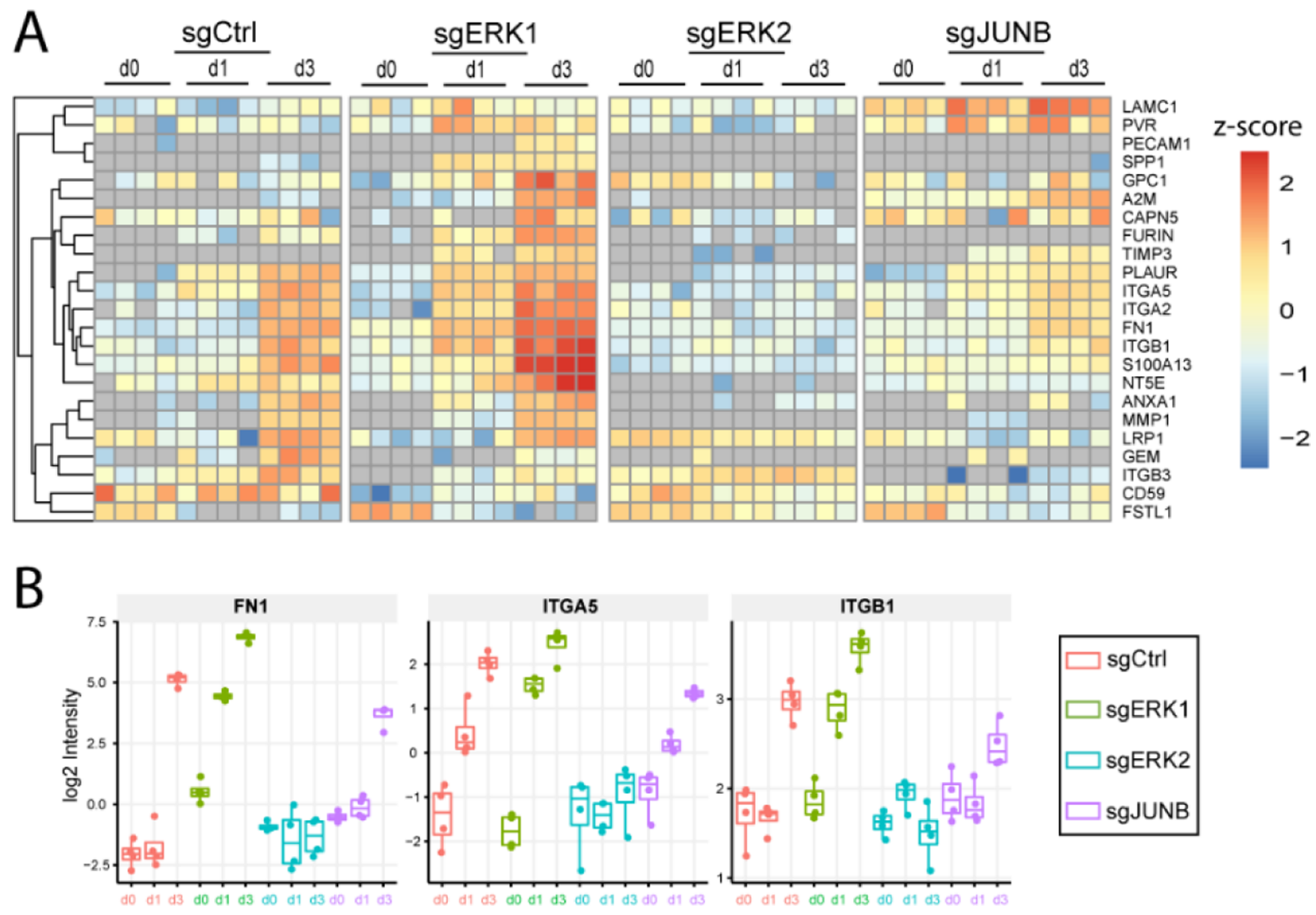

Figure 3. (A) Proteins enriched in EMT, missing values are represented in gray. (B) Box plots presenting three important EMT-related proteins FN1, ITGA5, and ITGB1 in control (red), sgERK1 (green), sgERK2 (blue), and sgJUNB (purple) cells.

Consistently, we found here that silencing of ERK2 maintains EMT-related proteins at a low expression level upon drug withdrawal (Figure 3A). However, an opposite phenomenon was observed in sgCtrl, sgERK1, and sgJUNB cells, that is, the EMT-related proteins were upregulated upon drug removal (Figure 3A). These include important EMT proteins, such as fibronectin (FN1), integrin $\beta$-1 (ITGB1), and integrin $\alpha-5$ (ITGA5) (Figure $3 \mathrm{~B}$ ). ${ }^{28} \mathrm{FN} 1$ is an established marker for EMT and has been linked to promote cancer growth, including in melanoma. ${ }^{29}$ ITGB1 was reported to enhance EMT via the FAK-AKT signaling pathway, ${ }^{30}$ and an increased expression of ITGB1 has been associated with breast tumor progression. ${ }^{31}$ Furthermore, ITGA5 has been shown to induce EMT transition and invasion in human cancer cells, after being cooperatively upregulated by twist 1 and AP- $1 .{ }^{32}$ Taking these data together shows that silencing of ERK2 results in no change or slight downregulation of proteins involved in EMT, yet ERK1 KO shows a strong increase in EMT-related proteins upon drug withdrawal, similar to the control and JUNB KO condition but slightly enhanced. These results suggest that EMT activation in drug-addicted melanoma cells upon drug withdrawal is affected by silencing ERK2 but not ERK1 or JUNB.

\section{Depletion of JUNB Shows a Similar Behavior as sgERK2}

Consistent with the t-SNE plot in Figure 1D, unsupervised hierarchical clustering of the Pearson correlations (1673 DE proteins) showed sgJUNB samples clustered together over time (i.e., d0, d1, and $\mathrm{d} 3$ ), where the correlation between biological replicates generally exceeded 0.9 (Figure S2A). More than half $(937 / 1673)$ of these proteins were upregulated upon drug withdrawal (Figure S2B,C). Among the upregulated clusters, C3 shows continuous upregulation after drug withdrawal, while $\mathrm{C} 4$ shows first downregulation of expression at day 1 and then amplification at day 3 (Figure S2B). Biological enrichment reveals proteins in $\mathrm{C} 3$ to be involved in the regulation of cytokine (interferon- $\alpha$ and TNF- $\alpha$ ) signaling, cell cycle (E2F targets and mitotic spindle) pathways, and mTORC1 signaling. Proteins in C4 are involved in cell division and interferon-gamma signaling (Figure S2D). Consistent with ERK2 knockout cells, the continuously downregulated proteins in cluster $\mathrm{C} 2$ regulate oxidative phosphorylation, apoptosis, and adipogenesis (Figure S2C,D).

The loss of JUNB in drug-addicted melanoma cells results in largely the same proteome response upon drug withdrawal as in the ERK2-depleted cells, showing amplification of the cell cycle and activation of the mTORC1 and cytokine pathway upon drug withdrawal, meanwhile, suppressing energy metabolism. As shown in Figure S2E, silencing of ERK2 shows more unique DE proteins, and this number increases upon drug withdrawal. Moreover, more common proteins between sgERK2 and sgJUNB cells were detected, which is more obvious over time upon drug withdrawal. In total, 528 differential proteins were in common between sgERK2 and sgJUNB upon drug withdrawal day 3, showing enrichment in, among others, cell cycle signaling (E2F targets and mitotic 

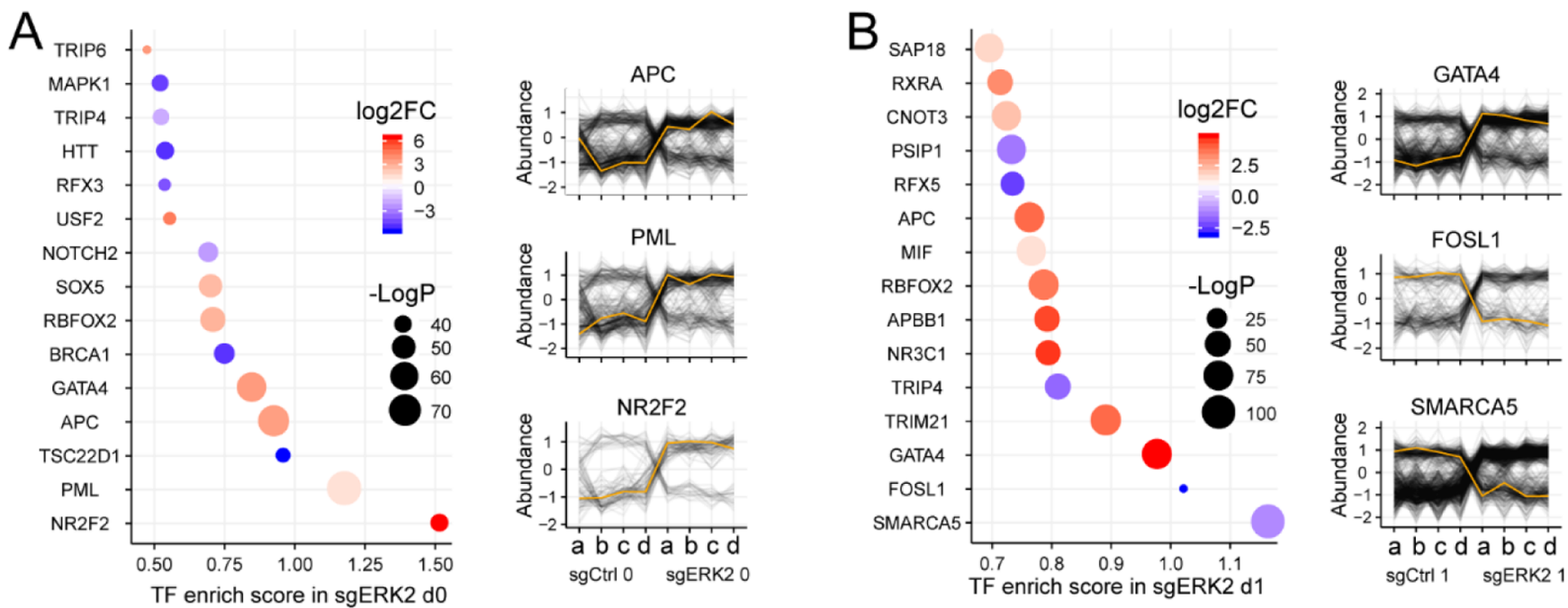

C
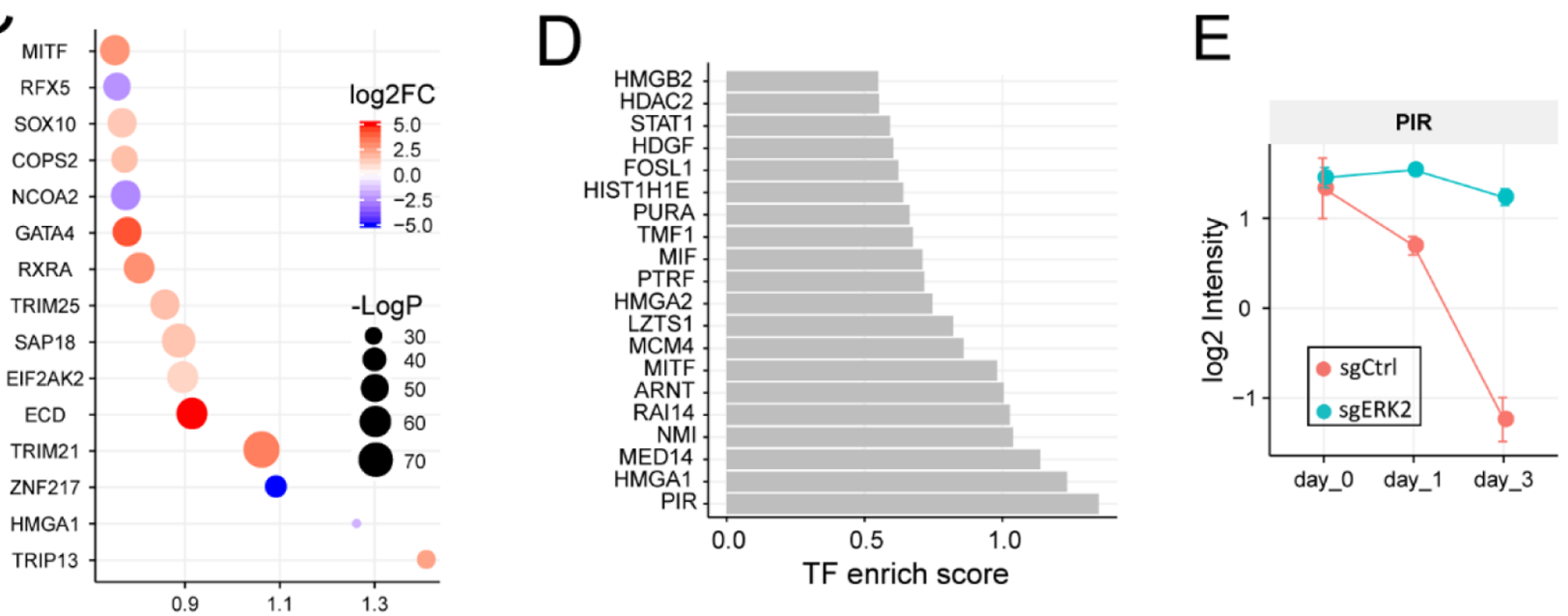

TF enrich score in sgERK2 d3
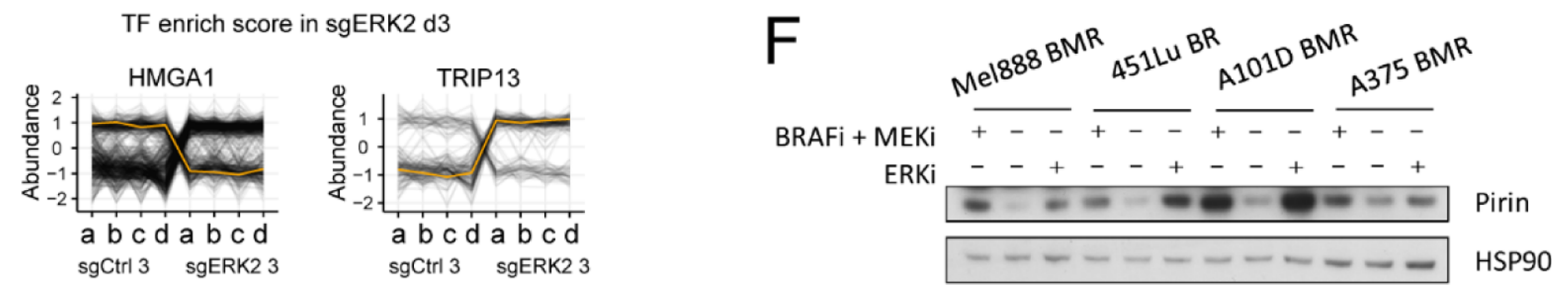

Figure 4. Transcription factor (regulator) enrichment. (A-C) (left) Top 15 significant transcription regulators enriched in sgERK2 on day 0 , day 1 , and day 3 separately, shades by the log2-fold change in expression intensity, and the $-\log p$-value of TF enrichment denotes dot size; (right) abundance of three TFs (highlighted in yellow line) with coexpression proteins (black lines). (D) Top significant TFs with pooled differentially changed proteins in sgERK2. (E) PIR (encode of pirin) expression intensity in sgCtrl (red) and sgERK2 (blue); the error bar presents \pm 1 SE. (F) Four drug-addicted melanoma cell line panels (BR for cells resistant to BRAFi and BMR for cells resistant to the combined BRAFi and MEKi) were cultured (1) with or without MAPK inhibitors and (2) with or without ERK inhibitors and then immunoblotted.

spindle) and oxidative phosphorylation (Figure S2F), suggesting a common phenotype where cells survive after drug withdrawal in combination with depletion of JUNB and ERK2 in melanoma cells.

\section{WGCNA Module-Based Transcription Factor (Regulator)} Enrichment

The RAS-RAF-MEK-ERK signaling pathway, directly and indirectly, interacts with transcription factors and their regulators, thereby controlling cell survival and proliferation. ${ }^{33}$ To systematically depict the underlying interconnectivity (e.g., interactions between proteins and transcription factors) in BRAFi-resistant melanoma cells, a module-based weighted protein (gene) coexpression network analysis (WGCNA) was carried out to explore correlations between DE proteins and regulators. $^{21}$ Next, a one-tailed hypergeometric test was used to determine the importance of highlighted transcription factors and regulators. ${ }^{34-36}$ An enrichment score for TFs was given by incorporating the exhibited significant differential expression $(p<0.05)$ and significant enrichment $(p<0.05)$ (see the Materials and Methods).

Figure 4A shows the top-scored transcription factors and regulators in ERK2 $\mathrm{KO}$ at d0 (with drug present). NR2F2, also known as COUP-TFII (chicken ovalbumin upstream promoter-transcription factor II), acts as a major angiogenesis regulator in the tumor microenvironment by regulating the 

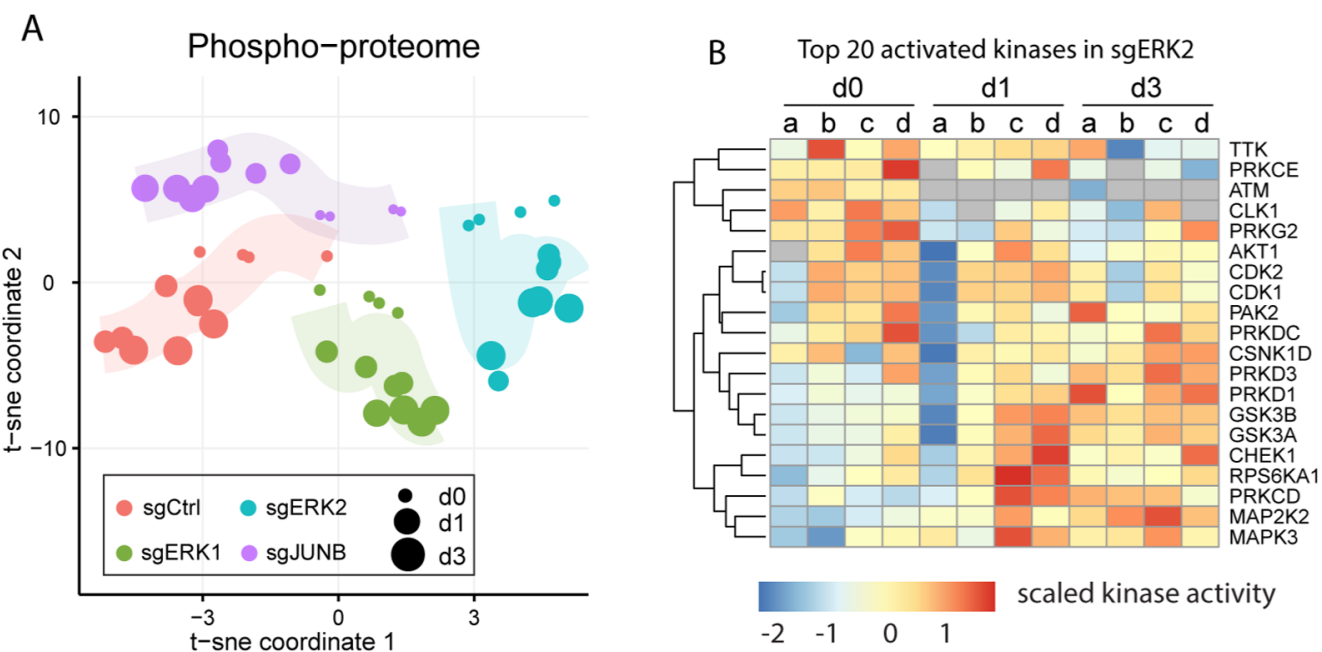

C
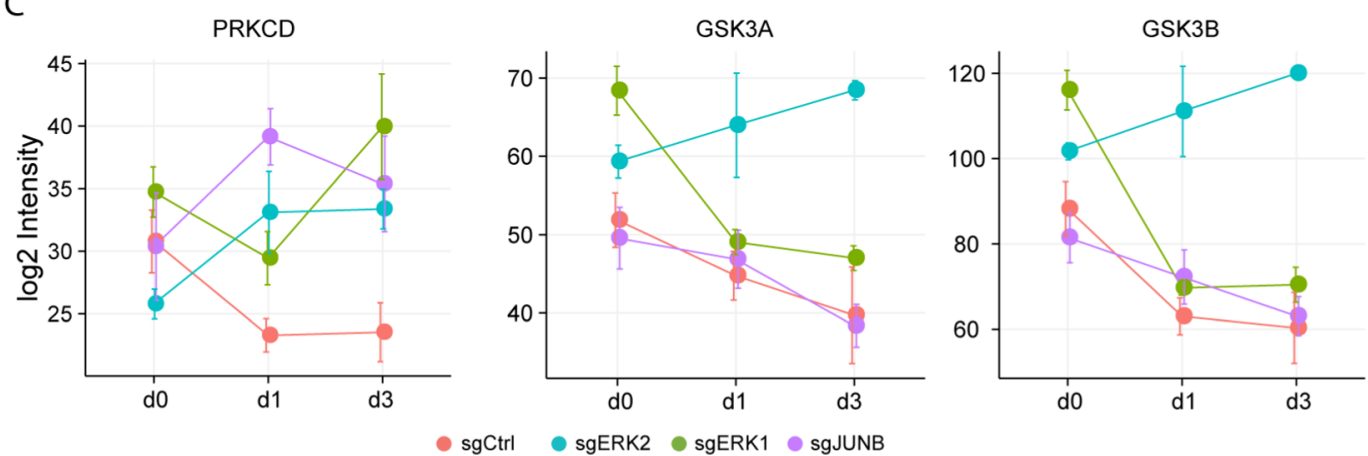

Figure 5. Phosphoproteome inferred kinase activity. (A) Phosphoproteomic profiling-based t-SNE plot shows the trajectory of how different cells proceeded upon drug removal. (B) Top 20 activated kinases in sgERK2. (C) Kinase activities in control (red), sgERK2 (blue), sgERK1 (green), and sgJUNB (purple); the error bar presents $\pm 1 \mathrm{SE}$.

transcription of angiopoietin- $1^{37}$ and promotes metastasis by the loss of miR-101 and miR-27a, thereby inducing FOXM1 and CENPF in prostate cancer. ${ }^{38}$ Furthermore, the top enriched regulators include tumor suppressor genes such as PML, APC, and GATA4, ${ }^{39-41}$ which are upregulated when the drug was present. For instance, the tumor suppressor PML is involved in regulating the $\mathrm{p} 53$ response to oncogenic signals and overexpression of PML induces senescence in a p53dependent manner. ${ }^{42}$ Knocking out PML impaired the p53regulatory pathway for apoptosis and the induction of p53 target genes such as Bax and p21 upon $\gamma$-irradiation. ${ }^{43}$

Next, we looked at the top 15 transcription factors showing high correlation with the DE proteins at $\mathrm{d} 1$ and $\mathrm{d} 3$ after drug removal (Figure 4B,C). Consistent with the results from Kong et al., ${ }^{12}$ the FOSL1 (encodes FRA1) induction after drug withdrawal was diminished in sgERK2 cells. Previous research showed that FOSL1 can act oncogenic to transform melanocytes, enabling subcutaneous tumor growth, through downregulation of MITF in an HMGA1-dependent manner. ${ }^{42}$ Interestingly, we observed a strong decrease in MITF abundance in sgCtrl and sgERK1 cells upon drug withdrawal, whereas a high expression level was maintained in sgERK2 and sgJUNB cells upon drug withdrawal (Figure S3). Conversely, HMGA1 (one of the coexpression proteins for MITF in our prediction result) showed an opposite trend compared to MITF, that is, upregulation in sgCtrl and sgERK1 cells, while maintaining a low abundance level in sgERK2 and sgJUNB cells. Furthermore, the transcription factor MITF was reported to be coregulated by SOX10, both of these two TFs show enrichment at $\mathrm{d} 3$. It has been shown that downregulation of SOX10 results in a simultaneous reduction of MITF and increased SOX9 expression, ${ }^{44}$ and a low level of MITF was detected in an "invasive" type of melanoma. ${ }^{45}$ The most significantly enriched transcription factors at $\mathrm{d} 1$ and $\mathrm{d} 3$ were SMARCA5 (also known as SNF2H) (Figure 4B) and TRIP13 (a key mitosis regulator) (Figure 4C), respectively. SMARCA5, involved in preventing genomic instability ${ }^{46}$ and interacting with the miR-99 family to regulate the DNA damage response, ${ }^{47}$ shows lower expression in sgERK2 upon drug withdrawal at d1. TRIP13 is reported to promote colorectal cancer progression by modulating EMT-related protein YWHAZ (14-3-3 protein zeta/delta). ${ }^{48}$

Compared to sgERK2, sgERK1 alters a completely different set of transcription factors and regulators (Figures S4A and 4B), mainly showing downregulation at $\mathrm{d} 1-\mathrm{d} 3$. The observed downregulation of many of these factors can be explained by the decreased cell viability at drug withdrawal. This can be illustrated by the downregulation of MCM proteins (MCM2, MCM3, and MCM5), which are involved in governing DNA replication and the cell cycle process. ${ }^{49}$ At $\mathrm{d} 2$, the strongest upregulation is observed for NAB2, a suppressor of the inducible zinc finger transcription factors EGR1 and EGR2, which regulate the expression of genes involved in differentiation, growth, and response to extracellular signals. ${ }^{50}$

Interestingly, silencing of JUNB and ERK2 displays a more common profile of significant transcription factors (regulators) 
(Figures S4C and 4). For example, the transcriptional repressor TSC22D1 (TSC22 domain family protein 1), ${ }^{51}$ which acts as a negative feedback regulator of Ras/Raf signaling, ${ }^{51}$ shows reduced expression in the presence of the drug in both sgERK2 and sgJUNB. In addition, changed expression of the chromatin remodeling factor HMGA1 was observed upon drug withdrawal. HMGA1 has been reported to function in melanocyte progression to melanoma and involved in EMT. Maurus et al. showed that siRNA-mediated reduction of HMGA1 partially prevented the FOSL1-mediated reduction of MITF on the RNA and protein level. ${ }^{52}$ Pegoraro et. al. demonstrated that knockdown of HMGA1 induces the mesenchymal-to-epithelial transition and dramatically decreases stemness and self-renewal in basal-like breast cancer. ${ }^{53}$

Next, we pooled our data over all three days, which results in PIR, which encodes pirin, a transcriptional coregulator of nuclear factor $(\mathrm{NF}) \kappa \mathrm{B}$, to be most significantly enriched in sgERK2 (Figure 4D). Pirin has been described to inhibit melanocytic cell senescence ${ }^{54}$ and regulate migration of melanoma cells. ${ }^{55}$ Previous research revealed that pirin can bind to $\mathrm{Bcl} 3$ that interacts with $\mathrm{NF} \kappa \mathrm{B}$, thereby enhancing cell survival, proliferation, and tumor malignancy. ${ }^{56-58}$ Pirin expresses stably upon drug withdrawal in sgERK2 cells, while it is strongly downregulated in sgCTRL cells (Figure 4E). Moreover, pirin expression is high in four different melanoma cells (e.g., Mel888 $8^{\mathrm{BMR}}, 451 \mathrm{Lu}^{\mathrm{BR}}, \mathrm{A} 101 \mathrm{D}^{\mathrm{BMR}}$, and $\mathrm{A} 375^{\mathrm{BMR}}$ ) treated with either a combination of BRAFi and MEKi or ERKi (Figure 4F). However, pharmacological inhibition of PIR failed to prevent lethality caused by drug removal in these BRAFiresistant cells. Next, we wondered whether restoring expression of pirin in control cells could reverse the observed cell death upon drug withdrawal. For this, we overexpressed PIR in BRAFi-resistant 451Lu and BRAFi + MEKi-resistant Mel888 cells (Figure S5). However, we did not observe such a rescue effect, suggesting that PIR acts downstream of ERK2 (being a biomarker rather than a functional mediator) and ERK2 KO prevents its degradation.

\section{Phosphoproteome Profiling Inferred Kinase Activity}

To further investigate the cellular signaling dynamics in these different melanoma cells, we performed phosphoproteome profiling, quantifying 22,117 phosphosites, 15,124 with a localization probability over 0.75 , which mapped to 4070 proteins (Table S2A). Similar to our proteome profile, the $t$ SNE plot shows that the different genetic clones followed distinct trajectories over time, while biological replicates group tightly together (Figure 5A). In our data, we observed the activating close-proximity phosphosites T202/Y204 on ERK1 and T185/Y187 on ERK2, 59 showing significant upregulation upon drug withdrawal under all conditions except in the conditions, where either of these genes were silenced (Figure S6).

To further unveil the role of the observed phosphorylation dynamics in these cells, an R pipeline "InKA", which integrates kinome, activation loop, phosphoSitePlus, and NetworKIN evidences, was applied to infer kinase activity. ${ }^{23}$ Based on this substrate-centric kinase activity prediction model, a total of 131 kinases were predicted with a relative activity score (Table S2B). We observed that ERK1, ERK2, and CDK1 are the most-activated kinases, which is more obvious over time upon drug withdrawal in control, sgERK1, and sgJUNB, indicating their dominant roles in drug-resistant melanoma. To obtain a high confident kinase activity, only those top 20 scoring kinases with an average score around 50 in each condition are presented (Figure S7A). Of the top 20 kinases, silencing of ERK2 results in the highest number of activated kinases upon drug removal, followed by silencing of JUNB. Moreover, several kinases were predicted to be activated upon drug withdrawal both in sgERK2 and sgJUNB cells (Figures 5B and S7C), including MAPK pathway members such as MAP2K2 (MEK2), AKT1, and MAPK3 (Figure S7B) and cell cycle kinases CDK1/2. Notably, no changes were detected in protein levels of MAP2K2 (MEK2), while in our phosphoproteome data, we observed a significant amplification in activity upon drug withdrawal. Conversely, most of those kinases show decreasing activity in sgERK1 and control cells when ceasing drug administration. These results suggest that silencing of ERK2 and JUNB shares more similarities in phosphorylation profiles, activating key members in MAPK and cell cycle signaling.

An interesting observation is the kinase PRKCD which has been reported to have contradicting roles in cell survival and death. ${ }^{60}$ The kinase is enriched under all conditions, however, shows maximum enrichment on day 1 of drug withdrawal in Erk2 and JunB KO cells, while its maximum is reached on day 3 in Erk1 KO cells (Figure 5C). However, the most striking difference observed in kinase activation is that of glycogen synthase kinase-3 (GSK3). We found GSK3A and GSK3B hyperactivated in ERK2 KO melanoma cells, while their activity decreased in the other three conditions upon drug withdrawal (Figures 5C and S7C). Previous research reveals that inhibition of ERK1/2 restores GSK3B activity and protein synthesis levels in a tuberous sclerosis model. ${ }^{61}$ Our data indicate that the control of GSK3B in drug-resistant melanoma cells is indeed controlled by ERK2 but not ERK1. It has been shown that the level of glycogen synthase kinase-3 (GSK3) in its active form is higher in tumor cells compared to normal tissue. $^{62}$ Furthermore, GSK3 is active downstream in both $\mathrm{PI} 3 \mathrm{~K}$ and Wnt pathways, which converges MAPK signaling to control MITF nuclear export, ${ }^{63}$ and promote cell survival and growth in human melanoma cells. ${ }^{64}$

\section{CONCLUSIONS}

Here, we present a proteomics and phosphoproteomics profiling of BRAFi-addicted melanoma cells (i.e., $451 \mathrm{Lu}$ cell line carries BRAF $^{\mathrm{MUT}}$ ) in response to BRAFi withdrawal. Silencing of ERK2 and JUNB could prevent drug addiction and reverse drug withdrawal-induced cell death, in contrast, inactivation of ERK1 failed to do so. Depletion of ERK2 and JUNB shares more similar proteome profiles upon drug withdrawal, while the proteome response in ERK1-depleted cells resembles that in control cells. Notably, we find a strong increase in EMT-related proteins upon drug withdrawal in both control and ERK1-depleted cells, which is abrogated by silencing of ERK2. These results suggest that EMT activation in drug-addicted melanoma cells upon drug withdrawal is affected by silencing ERK2 but not ERK1. Moreover, we identify PIR as an effector of ERK2 and show amplification of GSK3 kinase activity upon silencing of ERK2 but not ERK1. Our results depict how ERK1, ERK2, and JUNB influence the proteome response of drug-addicted melanoma cells upon drug withdrawal, which may help future strategies fighting drug resistance. 


\section{ASSOCIATED CONTENT}

\section{Supporting Information}

The Supporting Information is available free of charge at https://pubs.acs.org/doi/10.1021/acs.jproteome.1c00331.

Heat map of sample Pearson correlation in sgERK2 and sgERK1 cells; differential proteins in sgJUNB; box plot representing the intensity of three proteins FOSL1, MITF, and HMGA1 in control (red), sgERK1 (green), sgERK2 (blue), and sgJUNB (purple) cells; transcription factor (regulator) enrichment; overexpression of pirin in drug-resistant melanoma cells; phosphorylation of MAPK1 (ERK2) and MAPK3 (ERK1) in control (red), sgERK2 (blue), sgERK1 (green), and sgJUNB (purple) cells; kinase activities inferred from the phosphoproteome; proteome data sets and statistical test results; all proteins quantified; differentially changing proteins in sgERK2 compared to sgCtrl cells; differentially changing proteins in sgERK1 compared to sgCtrl cells; differentially changing proteins in sgJUNB compared to sgCtrl cells; phosphoproteome data sets and kinase activities inferred from the phosphoproteome; all quantified phosphorylation sites; and list of kinases and the corresponding activities inferred from the phosphoproteome (XLSX)

(XLSX)

\section{AUTHOR INFORMATION}

\section{Corresponding Author}

Maarten Altelaar - Biomolecular Mass Spectrometry and Proteomics Group, Utrecht Institute for Pharmaceutical Science, Utrecht University, Utrecht $3584 \mathrm{CH}$, The Netherlands; Netherlands Proteomics Center, $3584 \mathrm{CH}$ Utrecht, The Netherlands; 이이.org/0000-0001-50935945; Email: m.altelaar@uu.nl

\section{Authors}

Bohui Li - Biomolecular Mass Spectrometry and Proteomics Group, Utrecht Institute for Pharmaceutical Science, Utrecht University, Utrecht $3584 \mathrm{CH}$, The Netherlands; Netherlands Proteomics Center, $3584 \mathrm{CH}$ Utrecht, The Netherlands

Xiangjun Kong - Division of Molecular Oncology and Immunology, The Netherlands Cancer Institute, 1066 CX Amsterdam, The Netherlands

Harm Post - Biomolecular Mass Spectrometry and Proteomics Group, Utrecht Institute for Pharmaceutical Science, Utrecht University, Utrecht $3584 \mathrm{CH}$, The Netherlands; Netherlands Proteomics Center, $3584 \mathrm{CH}$ Utrecht, The Netherlands

Linsey Raaijmakers - Biomolecular Mass Spectrometry and Proteomics Group, Utrecht Institute for Pharmaceutical Science, Utrecht University, Utrecht $3584 \mathrm{CH}$, The Netherlands; Netherlands Proteomics Center, $3584 \mathrm{CH}$ Utrecht, The Netherlands

Daniel S. Peeper - Division of Molecular Oncology and Immunology, The Netherlands Cancer Institute, 1066 CX Amsterdam, The Netherlands

Complete contact information is available at:

https://pubs.acs.org/10.1021/acs.jproteome.1c00331

\section{Notes}

The authors declare no competing financial interest.

\section{ACKNOWLEDGMENTS}

This research was part of the Netherlands X-omics Initiative and partially funded by NWO (Project 184.034.019) and the China Scholarship Council (CSC) no. 201606300049.

\section{REFERENCES}

(1) Millington, G. W. M. Mutations of theBRAFgene in human cancer, by Davieset al. (Nature2002; 417: 949-54). Clin. Exp. Dermatol. 2013, 38, 222-223.

(2) Domingues, B.; Lopes, J.; Soares, P.; Populo, H. Melanoma treatment in review. ImmunoTargets Ther. 2018, 7, 35-49.

(3) Robert, C.; Grob, J. J.; Stroyakovskiy, D.; Karaszewska, B.; Hauschild, A.; Levchenko, E.; Chiarion Sileni, V.; Schachter, J.; Garbe, C.; Bondarenko, I.; Gogas, H.; Mandalá, M.; Haanen, J. B. A. G.; Lebbé, C.; Mackiewicz, A.; Rutkowski, P.; Nathan, P. D.; Ribas, A.; Davies, M. A.; Flaherty, K. T.; Burgess, P.; Tan, M.; Gasal, E.; Voi, M.; Schadendorf, D.; Long, G. V. Five-Year Outcomes with Dabrafenib plus Trametinib in Metastatic Melanoma. N. Engl. J. Med. 2019, 381, 626-636.

(4) Vido, M. J.; Le, K.; Hartsough, E. J.; Aplin, A. E. BRAF Splice Variant Resistance to RAF Inhibitor Requires Enhanced MEK Association. Cell Rep. 2018, 25, 1501-1510.

(5) Kozar, I.; Margue, C.; Rothengatter, S.; Haan, C.; Kreis, S. Many ways to resistance: How melanoma cells evade targeted therapies. Biochim. Biophys. Acta, Rev. Cancer 2019, 1871, 313-322.

(6) Shi, H.; Hugo, W.; Kong, X.; Hong, A.; Koya, R. C.; Moriceau, G.; Chodon, T.; Guo, R.; Johnson, D. B.; Dahlman, K. B.; Kelley, M. C.; Kefford, R. F.; Chmielowski, B.; Glaspy, J. A.; Sosman, J. A.; van Baren, N.; Long, G. V.; Ribas, A.; Lo, R. S. Acquired Resistance and Clonal Evolution in Melanoma during BRAF Inhibitor Therapy. Cancer Discovery 2014, 4, 80-93.

(7) Dietrich, P.; Kuphal, S.; Spruss, T.; Hellerbrand, C.; Bosserhoff, A. K. Wild-type KRAS is a novel therapeutic target for melanoma contributing to primary and acquired resistance to BRAF inhibition. Oncogene 2018, 37, 897-911.

(8) Stark, M. S.; Bonazzi, V. F.; Boyle, G. M.; Palmer, J. M.; Symmons, J.; Lanagan, C. M.; Schmidt, C. W.; Herington, A. C.; Ballotti, R.; Pollock, P. M.; Hayward, N. K. miR-514a regulates the tumour suppressor NF1 and modulates BRAFi sensitivity in melanoma. Oncotarget 2015, 6, 17753-17763.

(9) Zuo, Q.; Liu, J.; Huang, L.; Qin, Y.; Hawley, T.; Seo, C.; Merlino, G.; Yu, Y. AXL/AKT axis mediated-resistance to BRAF inhibitor depends on PTEN status in melanoma. Oncogene 2018, 37, 3275-3289.

(10) Irvine, M.; Stewart, A.; Pedersen, B.; Boyd, S.; Kefford, R.; Rizos, H. Oncogenic PI3K/AKT promotes the step-wise evolution of combination BRAF/MEK inhibitor resistance in melanoma. Oncogenesis 2018, 7, 72.

(11) Miller, M. A.; Oudin, M. J.; Sullivan, R. J.; Wang, S. J.; Meyer, A. S.; Im, H.; Frederick, D. T.; Tadros, J.; Griffith, L. G.; Lee, H.; Weissleder, R.; Flaherty, K. T.; Gertler, F. B.; Lauffenburger, D. A. Reduced Proteolytic Shedding of Receptor Tyrosine Kinases Is a Post-Translational Mechanism of Kinase Inhibitor Resistance. Cancer Discovery 2016, 6, 382-399.

(12) Kong, X.; Kuilman, T.; Shahrabi, A.; Boshuizen, J.; Kemper, K.; Song, J.-Y.; Niessen, H. W. M.; Rozeman, E. A.; Foppen, M. H. G.; Lank, C. U. B.; Peeper, D. S. Cancer drug addiction is relayed by an ERK2-dependent phenotype switch. Nature 2017, 550, 270.

(13) Moriceau, G.; Hugo, W.; Hong, A.; Shi, H.; Kong, X.; Yu, C. C.; Koya, R. C.; Samatar, A. A.; Khanlou, N.; Braun, J.; Ruchalski, K.; Seifert, H.; Larkin, J.; Dahlman, K. B.; Johnson, D. B.; Algazi, A.; Sosman, J. A.; Ribas, A.; Lo, R. S. Tunable-combinatorial mechanisms of acquired resistance limit the efficacy of BRAF/MEK cotargeting but result in melanoma drug addiction. Cancer Cell 2015, 27, 240256.

(14) Hong, A.; Moriceau, G.; Sun, L.; Lomeli, S.; Piva, M.; Damoiseaux, R.; Holmen, S. L.; Sharpless, N. E.; Hugo, W.; Lo, R. S. 
Exploiting Drug Addiction Mechanisms to Select against MAPKiResistant Melanoma. Cancer Discovery 2018, 8, 74-93.

(15) Guzmán, C.; Bagga, M.; Kaur, A.; Westermarck, J.; Abankwa, D. ColonyArea: an ImageJ plugin to automatically quantify colony formation in clonogenic assays. PLoS One 2014, 9, No. e92444.

(16) Post, H.; Penning, R.; Fitzpatrick, M. A.; Garrigues, L. B.; Wu, W.; MacGillavry, H. D.; Hoogenraad, C. C.; Heck, A. J. R.; Altelaar, A. F. M. Robust, Sensitive, and Automated Phosphopeptide Enrichment Optimized for Low Sample Amounts Applied to Primary Hippocampal Neurons. J. Proteome Res. 2017, 16, 728-737.

(17) Cox, J.; Mann, M. MaxQuant enables high peptide identification rates, individualized p.p.b.-range mass accuracies and proteome-wide protein quantification. Nat. Biotechnol. 2008, 26, 1367-1372.

(18) R Core Team. R: A Language and Environment for Statistical Computing; R Foundation for Statistical Computing: Vienna, Austria, 2017. https://www.R-project.org/.

(19) van der Maaten, L.; Hinton, G. Visualizing Data using t-SNE. J. Mach. Learn. Res. 2008, 9, 2579-2605.

(20) Zhou, Y.; Zhou, B.; Pache, L.; Chang, M.; Khodabakhshi, A. H.; Tanaseichuk, O.; Benner, C.; Chanda, S. K. Metascape provides a biologist-oriented resource for the analysis of systems-level datasets. Nat. Commun. 2019, 10, 1523.

(21) Tao, W.; Radstake, T. R.; Pandit, A. RegEnrich: An R package for gene regulator enrichment analysis reveals key role of ETS transcription factor family in interferon signaling. 2021, bioRxiv: 10.1101/2021.01.24.428029.

(22) Langfelder, P.; Horvath, S. WGCNA: an R package for weighted correlation network analysis. BMC Bioinf. 2008, 9, 559.

(23) Beekhof, R.; van Alphen, C.; Henneman, A. A.; Knol, J. C.; Pham, T. V.; Rolfs, F.; Labots, M.; Henneberry, E.; Le Large, T. Y.; Piersma, S. R.; Bertotti, A.; Trusolino, L.; Verheul, H. M.; Jimenez, C. R. INKA, an integrative data analysis pipeline for phosphoproteomic inference of active kinases. Mol. Syst. Biol. 2019, 15, No. e8981.

(24) Perez-Riverol, Y.; Csordas, A.; Bai, J.; Bernal-Llinares, M.; Hewapathirana, S.; Kundu, D. J.; Inuganti, A.; Griss, J.; Mayer, G.; Eisenacher, M.; Pérez, E.; Uszkoreit, J.; Pfeuffer, J.; Sachsenberg, T.; Yılmaz, Ş.; Tiwary, S.; Cox, J.; Audain, E.; Walzer, M.; Jarnuczak, A. F.; Ternent, T.; Brazma, A.; Vizcaíno, J. A. The PRIDE database and related tools and resources in 2019: improving support for quantification data. Nucleic Acids Res. 2019, 47, D442-D450.

(25) Kemper, K.; Krijgsman, O.; Kong, X.; Cornelissen-Steijger, P.; Shahrabi, A.; Weeber, F.; van der Velden, D. L.; Bleijerveld, O. B.; Kuilman, T.; Kluin, R. J. C.; Sun, C.; Voest, E. E.; Ju, Y. S.; Schumacher, T. N. M.; Altelaar, A. F. M.; McDermott, U.; Adams, D. J.; Blank, C. U.; Haanen, J. B.; Peeper, D. S. BRAF(V600E) Kinase Domain Duplication Identified in Therapy-Refractory Melanoma Patient-Derived Xenografts. Cell Rep. 2016, 16, 263-277.

(26) Kalluri, R.; Weinberg, R. A. The basics of epithelialmesenchymal transition. J. Clin. Invest. 2009, 119, 1420-1428.

(27) Lin, K.; Baritaki, S.; Militello, L.; Malaponte, G.; Bevelacqua, Y.; Bonavida, B. The Role of B-RAF Mutations in Melanoma and the Induction of EMT via Dysregulation of the NF-kappaB/Snail/RKIP/ PTEN Circuit. Genes Cancer 2010, 1, 409-420.

(28) Zhao, M.; Kong, L.; Liu, Y.; Qu, H. dbEMT: an epithelialmesenchymal transition associated gene resource. Sci. Rep. 2015, 5, 11459.

(29) Park, J.; Schwarzbauer, J. E. Mammary epithelial cell interactions with fibronectin stimulate epithelial-mesenchymal transition. Oncogene 2014, 33, 1649-1657.

(30) Ju, L.; Zhou, C. Integrin beta 1 enhances the epithelialmesenchymal transition in association with gefitinib resistance of nonsmall cell lung cancer. Cancer Biomarkers 2013, 13, 329-336.

(31) Yang, J.; Hou, Y.; Zhou, M.; Wen, S.; Zhou, J.; Xu, L.; Tang, X.; $\mathrm{Du}, \mathrm{Y}$.-e.; Hu, P.; Liu, M. Twist induces epithelial-mesenchymal transition and cell motility in breast cancer via ITGB1-FAK/ILK signaling axis and its associated downstream network. Int. J. Biochem. Cell Biol. 2016, 71, 62-71.
(32) Nam, E.-H.; Lee, Y.; Moon, B.; Lee, J. W.; Kim, S. Twist1 and AP-1 cooperatively upregulate integrin alpha5 expression to induce invasion and the epithelial-mesenchymal transition. Carcinogenesis 2015, 36, 327-337.

(33) Sánchez, I.; Hughes, R. T.; Mayer, B. J.; Yee, K.; Woodgett, J. R.; Avruch, J.; Kyriakls, J. M.; Zon, L. I. Role of SAPK/ERK kinase-1 in the stress-activated pathway regulating transcription factor c-Jun. Nature 1994, 372, 794-798.

(34) Ramirez, R. N.; El-Ali, N. C.; Mager, M. A.; Wyman, D.; Conesa, A.; Mortazavi, A. Dynamic Gene Regulatory Networks of Human Myeloid Differentiation. Cell Syst. 2017, 4, 416-429.

(35) Goode, D. K.; Obier, N.; Vijayabaskar, M. S.; Lie-A-Ling, M.; Lilly, A. J.; Hannah, R.; Lichtinger, M.; Batta, K.; Florkowska, M.; Patel, R.; Challinor, M.; Wallace, K.; Gilmour, J.; Assi, S. A.; Cauchy, P.; Hoogenkamp, M.; Westhead, D. R.; Lacaud, G.; Kouskoff, V.; Göttgens, B.; Bonifer, C. Dynamic Gene Regulatory Networks Drive Hematopoietic Specification and Differentiation. Dev. Cell 2016, 36, $572-587$.

(36) Langfelder, P.; Luo, R.; Oldham, M. C.; Horvath, S. Is My Network Module Preserved and Reproducible? PLoS Comput. Biol. 2011, 7, No. e1001057.

(37) Qin, J.; Chen, X.; Xie, X.; Tsai, M.-J.; Tsai, S. Y. COUP-TFII regulates tumor growth and metastasis by modulating tumor angiogenesis. Proc. Natl. Acad. Sci. U.S.A. 2010, 107, 3687-3692.

(38) Lin, S.-C.; Kao, C.-Y.; Lee, H.-J.; Creighton, C. J.; Ittmann, M. M.; Tsai, S.-J.; Tsai, S. Y.; Tsai, M.-J. Dysregulation of miRNAsCOUP-TFII-FOXM1-CENPF axis contributes to the metastasis of prostate cancer. Nat. Commun. 2016, 7, 11418.

(39) Salomoni, P.; Pandolfi, P. P. The role of PML in tumor suppression. Cell 2002, 108, 165-170.

(40) Muthusamy, V.; Duraisamy, S.; Bradbury, C. M.; Hobbs, C.; Curley, D. P.; Nelson, B.; Bosenberg, M. Epigenetic silencing of novel tumor suppressors in malignant melanoma. Cancer Res. 2006, 66, 11187-11193.

(41) Kang, C.; Xu, Q.; Martin, T. D.; Li, M. Z.; Demaria, M.; Aron, L.; Lu, T.; Yankner, B. A.; Campisi, J.; Elledge, S. J. The DNA damage response induces inflammation and senescence by inhibiting autophagy of GATA4. Science 2015, 349, aaa5612.

(42) Guo, A.; Salomoni, P.; Luo, J.; Shih, A.; Zhong, S.; Gu, W.; Paolo Pandolfi, P. The function of PML in p53-dependent apoptosis. Nat. Cell Biol. 2000, 2, 730-736.

(43) Pearson, M.; Carbone, R.; Sebastiani, C.; Cioce, M.; Fagioli, M.; Saito, S. i.; Higashimoto, Y.; Appella, E.; Minucci, S.; Pandolfi, P. P.; Pelicci, P. G. PML regulates p53 acetylation and premature senescence induced by oncogenic Ras. Nature 2000, 406, 207-210.

(44) Shakhova, O.; Zingg, D.; Schaefer, S. M.; Hari, L.; Civenni, G.; Blunschi, J.; Claudinot, S.; Okoniewski, M.; Beermann, F.; MihicProbst, D.; Moch, H.; Wegner, M.; Dummer, R.; Barrandon, Y.; Cinelli, P.; Sommer, L. Sox10 promotes the formation and maintenance of giant congenital naevi and melanoma. Nat. Cell Biol. 2012, 14, 882-890.

(45) Verfaillie, A.; Imrichova, H.; Atak, Z. K.; Dewaele, M.; Rambow, F.; Hulselmans, G.; Christiaens, V.; Svetlichnyy, D.; Luciani, F.; Van den Mooter, L.; Claerhout, S.; Fiers, M.; Journe, F.; Ghanem, G.-E.; Herrmann, C.; Halder, G.; Marine, J.-C.; Aerts, S. Decoding the regulatory landscape of melanoma reveals TEADS as regulators of the invasive cell state. Nat. Commun. 2015, 6, 6683.

(46) Toiber, D.; Erdel, F.; Bouazoune, K.; Silberman, D. M.; Zhong, L.; Mulligan, P.; Sebastian, C.; Cosentino, C.; Martinez-Pastor, B.; Giacosa, S.; D’Urso, A.; Näär, A. M.; Kingston, R.; Rippe, K.; Mostoslavsky, R. SIRT6 recruits SNF2H to DNA break sites, preventing genomic instability through chromatin remodeling. Mol. Cell 2013, 51, 454-468.

(47) Mueller, A. C.; Sun, D.; Dutta, A. The miR-99 family regulates the DNA damage response through its target SNF2H. Oncogene 2013, 32, 1164-1172.

(48) Sheng, N.; Yan, L.; Wu, K.; You, W.; Gong, J.; Hu, L.; Tan, G.; Chen, H.; Wang, Z. TRIP13 promotes tumor growth and is associated 
with poor prognosis in colorectal cancer. Cell Death Discovery 2018,9, 402.

(49) Evrin, C.; Clarke, P.; Zech, J.; Lurz, R.; Sun, J.; Uhle, S.; Li, H.; Stillman, B.; Speck, C. A double-hexameric MCM2-7 complex is loaded onto origin DNA during licensing of eukaryotic DNA replication. Proc. Natl. Acad. Sci. U.S.A. 2009, 106, 20240-20245.

(50) Kumbrink, J.; Kirsch, K. H.; Johnson, J. P. EGR1, EGR2, and EGR3 activate the expression of their coregulator NAB2 establishing a negative feedback loop in cells of neuroectodermal and epithelial origin. J. Cell. Biochem. 2010, 111, 207-217.

(51) Kester, H. A.; Blanchetot, C.; den Hertog, J.; van der Saag, P. T.; van der Burg, B. Transforming growth factor- $\beta$-stimulated clone22 is a member of a family of leucine zipper proteins that can homoand heterodimerize and has transcriptional repressor activity. J. Biol. Chem. 1999, 274, 27439-27447.

(52) Maurus, K.; Hufnagel, A.; Geiger, F.; Graf, S.; Berking, C.; Heinemann, A.; Paschen, A.; Kneitz, S.; Stigloher, C.; Geissinger, E.; Otto, C.; Bosserhoff, A.; Schartl, M.; Meierjohann, S. The AP-1 transcription factor FOSL1 causes melanocyte reprogramming and transformation. Oncogene 2017, 36, 5110-5121.

(53) Pegoraro, S.; Ros, G.; Piazza, S.; Sommaggio, R.; Ciani, Y.; Rosato, A.; Sgarra, R.; Del Sal, G.; Manfioletti, G. HMGA1 promotes metastatic processes in basal-like breast cancer regulating EMT and stemness. Oncotarget 2013, 4, 1293-1308.

(54) Licciulli, S.; Luise, C.; Scafetta, G.; Capra, M.; Giardina, G.; Nuciforo, P.; Bosari, S.; Viale, G.; Mazzarol, G.; Tonelli, C.; Lanfrancone, L.; Alcalay, M. Pirin inhibits cellular senescence in melanocytic cells. Am. J. Pathol. 2011, 178, 2397-2406.

(55) Miyazaki, I.; Simizu, S.; Okumura, H.; Takagi, S.; Osada, H. A small-molecule inhibitor shows that pirin regulates migration of melanoma cells. Nat. Chem. Biol. 2010, 6, 667-673.

(56) Dechend, R.; Hirano, F.; Lehmann, K.; Heissmeyer, V.; Ansieau, S.; Wulczyn, F. G.; Scheidereit, C.; Leutz, A. The Bcl-3 oncoprotein acts as a bridging factor between NF- $\kappa \mathrm{B} /$ Rel and nuclear co-regulators. Oncogene 1999, 18, 3316-3323.

(57) Park, S. G.; Chung, C.; Kang, H.; Kim, J.-Y.; Jung, G. Upregulation of cyclin $\mathrm{D} 1$ by $\mathrm{HBx}$ is mediated by NF- $\kappa \mathrm{B} 2 / \mathrm{BCL} 3$ complex through $\kappa \mathrm{B}$ site of cyclin D1 promoter. J. Biol. Chem. 2006, 281, 31770-31777.

(58) Courtois, G.; Gilmore, T. D. Mutations in the NF-kappaB signaling pathway: implications for human disease. Oncogene 2006, 25, 6831-6843.

(59) Goetz, E. M.; Ghandi, M.; Treacy, D. J.; Wagle, N.; Garraway, L. A. ERK mutations confer resistance to mitogen-activated protein kinase pathway inhibitors. Cancer Res. 2014, 74, 7079-7089.

(60) Kronfeld, I.; Kazimirsky, G.; Lorenzo, P. S.; Garfield, S. H.; Blumberg, P. M.; Brodie, C. Phosphorylation of protein kinase Cdelta on distinct tyrosine residues regulates specific cellular functions. $J$. Biol. Chem. 2000, 275, 35491-35498.

(61) Pal, R.; Bondar, V. V.; Adamski, C. J.; Rodney, G. G.; Sardiello, M. Inhibition of ERK1/2 Restores GSK3beta Activity and Protein Synthesis Levels in a Model of Tuberous Sclerosis. Sci. Rep. 2017, 7, 4174.

(62) Shakoori, A.; Ougolkov, A.; Yu, Z. W.; Zhang, B.; Modarressi, M. H.; Billadeau, D. D.; Mai, M.; Takahashi, Y.; Minamoto, T. Deregulated GSK3beta activity in colorectal cancer: its association with tumor cell survival and proliferation. Biochem. Biophys. Res. Commun. 2005, 334, 1365-1373.

(63) Ngeow, K. C.; Friedrichsen, H. J.; Li, L.; Zeng, Z.; Andrews, S.; Volpon, L.; Brunsdon, H.; Berridge, G.; Picaud, S.; Fischer, R.; Lisle, R.; Knapp, S.; Filippakopoulos, P.; Knowles, H.; Steingrímsson, E.; Borden, K. L. B.; Patton, E. E.; Goding, C. R. BRAF/MAPK and GSK3 signaling converges to control MITF nuclear export. Proc. Natl. Acad. Sci. U.S.A. 2018, 115, E8668-E8677.

(64) Kubic, J. D.; Mascarenhas, J. B.; Iizuka, T.; Wolfgeher, D.; Lang, D. GSK-3 promotes cell survival, growth, and PAX3 levels in human melanoma cells. Mol. Cancer Res. 2012, 10, 1065-1076. 